\title{
Publisher Correction: Universal elastic mechanism for stinger design
}

Kaare H. Jensen (D), Jan Knoblauch, Anneline H. Christensen, Katrine S. Haaning and Keunhwan Park (D)

Correction to: Nature Physics https://doi.org/10.1038/s41567-020-0930-9, published online 15 June 2020.

In the version of this Article originally published online, in Fig. 3 and its caption, the relation ' $F_{\mathrm{c}}<F<F_{\mathrm{f}}$ ' was incorrect; it should have been ' $F_{\mathrm{f}}<F<F_{\mathrm{c}}$ '. Also in Fig. 3, typographical errors led to missing minus signs from the superscripts on both axes; the vertical axis label was ' $F \times k_{2} a^{2} E^{1 / 3}\left(\mu p_{0}\right)^{4 / 3}$ ' but should have been ' $F \times k_{2} a^{-2} E^{1 / 3}\left(\mu p_{0}\right)^{-4 / 3}$, the horizontal axis label was ' $d_{0} / L \times k_{1} E^{1 / 3}\left(\mu p_{0}\right)^{1 / 3}$ ' but should have been ' $d_{0} / L \times k_{1} E^{1 / 3}\left(\mu p_{0}\right)^{-1 / 3}$ '. All versions of the Article have been amended.

Published online: 15 July 2020

https://doi.org/10.1038/s41567-020-0996-4

๑ The Author(s), under exclusive licence to Springer Nature Limited 2020 